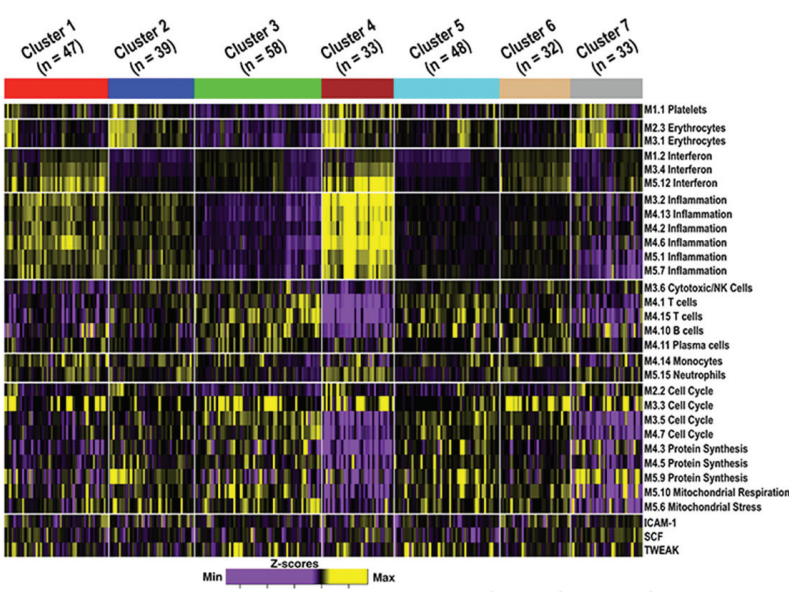

Abstract SAT0423 - Figure 1. Modular transcriptional score profiles of seven SLE clusters

Conclusions: SLE subsets can be distinguished by a range of molecular profiles encompassing IFN, T cell, neutrophil, plasmablast, and inflammation co-expression signatures, as well as soluble mediators that vary with disease activity. Prospective longitudinal studies of these molecular profiles may inform clinical trial design and personalised disease management.

Acknowledgements: This work was supported in part by grants from the National Institutes of Health: U19AI082714, U01Al101934, U54GM104938, and P30AR053483.

Disclosure of Interest: None declared

DOI: 10.1136/annrheumdis-2018-eular.6054

\section{SAT0424 COMPLEMENT C4 GENE COPY NUMBER VARIATIONS BESTOW LARGE RANGES OF SERUM C4 PROTEIN LEVELS IN CHINESE PATIENTS WITH SYSTEMIC LUPUS ERYTHEMATOSUS (SLE) AND CONTRIBUTE TO ORGAN AND CARDIOVASCULAR DAMAGES OVER TIME}

C.C. Mok ${ }^{1}$, E. King ${ }^{2}$, B. Zhou ${ }^{2}$, G. Yu ${ }^{2}$, Y.L. Wu ${ }^{2}$, C.Y. Yu ${ }^{2} .{ }^{1}$ Medicine, Tuen Mun Hospital, HK, Hong Kong, ${ }^{2}$ Pediatrics, Nationwide Children's Hospital, Columbus, Ohio, USA

Background: Human SLE is characterised by fluctuating serum levels of complement proteins. There are frequent copy number variations (CNVs) of complement $\mathrm{C} 4 \mathrm{~A}$ and $\mathrm{C} 4 \mathrm{~B}$ genes among different individuals. Previously, we demonstrated that $\mathrm{C} 4 \mathrm{~A}$ deficiency is a strong genetic risk factor for SLE.

Objectives: To investigate how CNVs of $\mathrm{C} 4$ contribute to the great variability of $\mathrm{C} 4$ serum levels and how deficiencies of $\mathrm{C} 4 \mathrm{~A}$ or $\mathrm{C} 4 \mathrm{~B}$ modulate the clinical presentations, including organ damage, of SLE.

Methods: Our study population included 499 patients from Hong Kong, who fulfilled $\geq 4$ of the 2013 ACR/SLICC criteria for SLE. Among them 93\% were women, the mean age of SLE onset was $32.8 \pm 13.0$ years, and SLE duration was $14.4 \pm 7.6$ years. Gene copy numbers (GCNs) of total C4 (C4T), C4A and C4B were determined by real-time PCRs. Serial serum levels over the past 5 years for C4 and C3 of each patient were retrieved through the laboratory data registry system. Serum C4 and C3 levels are shown as mg/100 ml (unit). Clinical manifestations and organ damage of SLE were correlated with CNVs of C4 genes and serum levels. Continuous data between groups were compared by t-tests and categorical data by $\chi 2$ analyses. Logistic regression was used to calculate odds ratios (ORs) and 95\% confidence intervals for effects of C4 CNVs on cumulative clinical manifestations of SLE and accrued organ damage, adjusted for durations of disease.

Results: Serum levels for C4 varied from 1-84 units (Median: 17) and for C3 from 8-314 units (Median: 86). There was a very strong correlation between C4 and C3 protein levels $\left(R=0.70, p=5.3 \times 10-{ }^{75}\right)$. The GCN of C4T varied between 2 and 9 with a median of 4 copies (54\%), followed by 2 and 3 copies $(21 \%)$. Each additional gene copy correlated to an increase of 4 and 6 units for the mean and maximum serum C4 levels, respectively. A higher GCN of C4T $(\geq 3$ vs $<3)$ was protective against the development of neuropsychiatric disorder over time [OR $0.45(0.21-0.98), p=0.04]$. A high GCN of C4L ( $\geq 3$ vs $<3)$, or the absence of C4S $(\mathrm{GCN}=0)$, was negatively associated with the occurrence of thrombocytopenia
[OR $0.64(0.42-0.97), p=0.04]$. A high GCN of $C 4 B$ was associated with damage to any organ [OR $1.76(1.05-2.93), p=0.03$ ], but a high $\mathrm{GCN}$ of $\mathrm{C} 4 \mathrm{~A}(\geq 3 \mathrm{vs}<3$ ) was associated with cardiovascular damage [OR $2.30(1.06-5.00), p=0.04]$. Among the SLE patients studied, $18.3 \%$ had persistently low levels of $\mathrm{C} 4$ (mean $\leq 10.0$ units). These patients mostly had GCNs of C4T=2 or 3 [OR 4.02 (2.47-6.56), $\mathrm{p}=4.7 \times 10^{8}-^{8}$ ], or $\mathrm{C} 4 \mathrm{~B}=0$ or 1 [OR $3.06(1.89-4.96), \mathrm{p}=9.0 \times 10^{-6}$ ]. Patients with persistently low $\mathrm{C} 4$ levels had increased prevalence of mucosal ulceration [OR 2.09 (1.15-3.78), $p=0.02$ ], lymphopenia [OR $1.76(1.01-3.05)$, $\mathrm{p}=0.045$ ] and gastrointestinal disorders [OR 2.52 (1.31-4.84), $\mathrm{p}=0.005$ ].

Conclusions: CNVs of C4 genes confer great variability of serum C4 levels among SLE patients. While C4A deficiency contributes to genetic predisposition of SLE, persistently low levels of serum C4 among patients were strongly correlated with low GCN of total $\mathrm{C} 4$ and $\mathrm{C} 4 \mathrm{~B}$ deficiency. Elucidating C4-CNVs may have prognostic significance of SLE as high GCNs of C4B and C4A appeared to correlate with organ damage and cardiovascular disease, respectively.

Disclosure of Interest: None declared

DOI: 10.1136/annrheumdis-2018-eular.4974

\section{SAT0425 PHENOTYPING PRIMARY SJÖGREN'S SYNDROME (PSS) BY USING SALIVARY GLAND ULTRASONOGRAPHY: A STEP FORWARD TO INDIVIDUALISED THERAPIES}

C. Baldini ${ }^{1}$, F. Ferro ${ }^{1}$, N. Luciano ${ }^{1}$, E. Calabresi ${ }^{1}$, E. Elefante ${ }^{1}$, V. Donati ${ }^{2}$, M. Mosca ${ }^{1}$, S. Bombardieri' ${ }^{1}{ }^{1}$ Rheumatology Unit, University of Pisa; ${ }^{2}$ Pathologic Anatomy II, Pisa, Italy

Background: Salivary gland ultrasonography (SGUS) has recently appeared as a useful tool to non invasively assess major salivary glands inflammation and damage in primary Sjögren's syndrome (pSS). To date, relatively few studies have evaluated the usefulness of SGUS in characterising different disease phenotypes ultimately providing information on which patients might be most likely to benefit from specific interventions

Objectives: to explore whether SGUS may be useful in identifying different clinical-serological and histopathological phenotypes of pSS, ultimately allowing individualised treatments.

Methods: Sonographic data were retrieved from a large monocentric cohort of pSS patients. The echostructure of each gland on B-mode images was graded on a 5 -point scale (0-4), and a SGUS score $\geq 2$ was defined as pathological. Hypoanechoic areas in the glands were defined as isolated $(<25 \%$ of the surface), localised (25\%-50\%) and diffuse-scattered (>50\%). The biggest hypo or anechoic area in each gland was measured. SGUS was repeated in 150 pSS patients at least twice over a median follow up of 24 months. All the patients received a complete rheumatological assessment: disease activity was measured by the EULAR Sjögren's Syndrome Disease Activity Index (ESSDAI), histopathological evaluation included the assessment of the focus score (FS) and the reporting of the number of germinal centre (GC)-like structures.

Results: We included in this study 311 patients $(300 \mathrm{~F}: 11 \mathrm{M}$; median age $=58$ years (IQR, 49-69)) and median disease duration of 3 years (IQR 0-9). Out of them $136 / 311(43.7 \%)$ had a SGUS score $<2$ with none or isolated hypo-anechoic areas in their glands. Localised areas were detected in 87/311 (28\%) pSS patients and diffuse-scattered areas in $88 / 311(28.3 \%)$. Patients with none or isolated hypo-anechoic areas tended to be older $(p=0.06)$ and presented a significantly lower FS, a lower ESSDAI and a higher unstimulated salivary flow rate when compared to the other pSS patients $(p<0.001)$. Patients with localised or diffuse-scattered echographic pattern were more frequently seropositive for anti-Ro/SSA anti-La/SSB and rheumatoid factor, presented more frequently hypergammaglobulinemia and low $\mathrm{C} 3 / \mathrm{C} 4$ levels and had a higher frequency of GC-like structures in their biopsies $(p<0.001)$. Patients with diffuse-scattered hypo-anechoic areas presented the highest disease activity, in particular in the ESSDAI glandular, cutaneous and haematological domains. Notably, the SGUS score did not change over the time in patients who underwent repeated SGUS evaluations. Out of 136 patients with a SGUS score $<2,58$ underwent additional SGUS evaluations over the follow-up. The SGUS was unchanged in all the cases and still associated with a low systemic disease activity.

Conclusions: This study highlighted the potential usefulness of SGUS in phenotyping pSS subsets. Patients with none or isolated hypoechoic areas in their glands are likely to present a low systemic disease activity during the disease course, being therefore candidate to less aggressive treatments. By contrast, patients with highest SGUS scores tend to present a more active systemic involvement and are candidate to receive a more aggressive therapy.

Disclosure of Interest: None declared

DOI: 10.1136/annrheumdis-2018-eular.5948 